\title{
Diffusion-Weighted Echo Planar Imaging using MUltiplexed Sensitivity Encoding and Reverse Polarity Gradient in Head and Neck Cancer: An Initial Study
}

\section{Amaresha Shridhar Konar', Maggie Fung' ${ }^{2}$, Ramesh Paudyal', Jung Hun Oh', Yousef Mazaheri ${ }^{1,3}$, Vaios Hatzoglou ${ }^{3}$, and Amita Shukla-Dave ${ }^{1,3}$}

${ }^{1}$ Department of Medical Physics, Memorial Sloan Kettering Cancer Center, New York, NY; ${ }^{2}$ GE Healthcare, New York, NY; and ${ }^{3}$ Department of Radiology, Memorial Sloan Kettering Cancer Center, New York, NY

\section{Corresponding Author:}

Amita Shukla-Dave, PhD, FISMRM

Departments of Medical Physics and Radiology, Memorial Sloan Kettering Cancer Center, 1275 York Avenue, New York, New York 10065-6007

E-mail: davea@mskcc.org
Key Words: Head and neck region, diffusion-weighted MRI, echo planar imaging, eddy current, magnetic field inhomogeneity, geometrical distortion, multiplexed sensitivity encoding, reverse-polarity gradients

Abbreviations: head and neck $(H N)$, magnetic resonance imaging (MRI), diffusionweighted echo planar imaging (DW-EPI), apparent diffusion coefficient (ADC), singleshot-DWEPI (SS-DWEPI), field of view (FOV), echo time (TE), multiplexed sensitivity encoding (MUSE), sensitivity encoding (SENSE), reverse-polarity gradient (RPG), American College of Radiology (ACR), Quantitative Imaging Biomarker Alliance (QIBA), National Institute of Standards and Technology (NIST), Radiological Society of North America (RSNA), polyvinylpyrrolidone (PVP), Health Insurance Portability and Accountability Act (HIPAA), repetition time (TR), band width (BW), number of excitation (NEX), echo train length (ETL), array coil spatial sensitivity encoding (ASSET), control variable (CV), control points (CP), root-mean-square error (RMSE), region of interest (ROI), correlation coefficient (CC), Wilcoxon signed-rank (WSR), Kruskal-Wallis (KW)

We aimed to compare the geometric distortion (GD) correction performance and apparent diffusion coefficient (ADC) measurements of single-shot diffusion-weighted echo-planar imaging (SS-DWEPI), multiplexed sensitivity encoding (MUSE)-DWEPI, and MUSE-DWEPI with reverse-polarity gradient (RPG) in phantoms and patients. We performed phantom studies at 3T magnetic resonance imaging (MRI) using the American College of Radiology phantom and Quantitative Imaging Biomarker Alliance DW-MRI ice-water phantom to assess GD and effect of distortion in the measurement of ADC, respectively. Institutional review board approved the prospective clinical component of this study. DW-MRI data were obtained from 11 patients with head and neck cancer using these three DW-MRI methods. Wilcoxon signed-rank (WSR) and KruskalWallis $(\mathrm{KW})$ tests were used to compare ADC values, and qualitative rating by radiologist between three DWMRI methods. In the ACR phantom, GD of $0.17 \%$ was observed for the $b=0 \mathrm{~s} / \mathrm{mm}^{2}$ image of the MUSE-DWEPI with RPG method compared with that of $1.53 \%$ and $2.1 \%$ of MUSE-DWEPI and SS-DWEPI, respectively; The corresponding methods root-mean-square errors were $0.58,3.37$, and $5.07 \mathrm{~mm}$. WSR and KW tests showed no significant difference in the ADC measurement between these three DW-MRI methods for both healthy masseter muscles and neoplasms $(P>.05)$. We observed improvement in spatial accuracy for MUSE-DWEPI with RPG in the head and neck region with a higher correlation $\left(\mathbb{R}^{2}=0.791\right)$ compared with that for SS-DWEPI $\left(\mathbb{R}^{2}=0.707\right)$ and MUSE-DWEPI $\left(\mathbb{R}^{2}=0.745\right)$. MUSE-DWEPI with RPG significantly reduces the distortion compared with MUSE-DWEPI or conventional SS-DWEPI techniques, and the ADC values were similar.

\section{INTRODUCTION}

Head and neck (HN) cancer is one of the most common types of cancer and results in $>300000$ deaths per year worldwide (1). HN malignancies are heterogeneous, and tumor locations affect a variety of sites, including skin, skull base, salivary glands, paranasal sinuses, cervical aerodigestive tract, and thyroid gland $(2,3)$. Accurate diagnosis, staging, assessment of treatment response, and follow-up are crucial for patient management and are frequently accomplished by using magnetic resonance imaging (MRI) (4-7). 
Standard MRI provides high-resolution anatomical images, whereas quantitative MRI techniques such as diffusion-weighted (DW)-MRI measure the Brownian motion of water molecules in tumor tissue, which is highly reflective of cellular organization and membrane integrity (8). The apparent diffusion coefficient (ADC) derived from DW-MRI is a surrogate biomarker of tissue cellularity (9). DW-MRI has shown promise in characterizing HN lesions as either benign or malignant (10-14). ADC is a useful prognostic and predictive biomarker in HN cancers $(4,15-23)$ and can help determine whether a posttreatment mass represents a scar tissue or a residual/recurrent tumor (24-27). A recent review summarized the DW-MRI techniques, applications, and its limitations in HN cancer (28).

Single-shot diffusion-weighted echo planar imaging (SSDWEPI) is a commonly used DW-MRI acquisition method in the clinical setting because of its ability to acquire data with a relatively short scan time but is limited by lower resolution owing to off-resonance effects (29-31). Echo planar imaging (EPI) inherently suffers from spatial distortion because of residual magnetic field inhomogeneity caused by tissue susceptibility and spatial $\mathrm{B}_{0}$ inhomogeneity of the magnet in a large field of view (FOV) and can limit clinical imaging evaluation $(28,32,33)$. The magnetic field inhomogeneities cause pixels to shift spatially, and the effect is much more prominent in phase direction than in readout and slice-encoding direction (32). The shift in pixel location in the phase direction, $\delta \mathrm{y}$, can be characterized as follows:

$$
\delta \mathrm{y}(\mathrm{x}, \mathrm{y}, \mathrm{z})=\left(t_{\text {esp }}\right) \delta B(x, y, z) / \tau G
$$

where $t_{\mathrm{esp}}$ is the echo spacing (the length of time between the start of each readout line), $\tau$ is the time for each phase-encoding period, $\delta B(x, y, z)$ is the $\mathrm{B}_{0}$ inhomogeneity at location $(x, y, z)$, and $G$ is the average phase-encoding gradient amplitude (34).

Incremental changes have been made in DW-MRI acquisition, such as interleaved multishot EPI techniques, which uses short echo train length per shot and short echo time (TE), thereby reducing the distortion (35-40). The multiplexed sensitivityencoding (MUSE) method (41) uses the conventional sensitivity encoding (SENSE) technique (42) to measure the motion-induced phase variations among multiple shots and then performs joint unaliasing from all the shots. Separate navigator echoes are not required, avoiding the pitfall of motion inconsistency between the navigator echoes and SS-EPI acquisition. MUSE-DWEPI has shown its ability to reduce geometric distortion (GD) in comparison with SS-DWEPI in both the American College of Radiology (ACR) phantom and the healthy human brain $(41,43)$.

In addition to MUSE-DWEPI, the reverse-polarity gradient (RPG) method (a postprocessing technique) has been used for further distortion correction $(34,44)$. The RPG technique acquires an additional $\mathrm{b}=0 \mathrm{~s} / \mathrm{mm}^{2}$ image set with opposite phase-encoding polarities. The image set will have precisely the same but opposite spatial distortion pattern compared with the reference $b=0 \mathrm{~s} / \mathrm{mm}^{2} \mathrm{DW}$ MRI image. MUSE-DWEPI with RPG has successfully been tested for brain (34) and prostate in clinical applications (45). To the best of our knowledge, there is no study conducted on HN region using MUSE-DWEPI with RPG to address spatial distortions. The present study aims to compare the distortion-correction performance and ADC values obtained from SS-DWEPI, MUSE-DWEPI, and MUSEDWEPI with RPG methods in phantoms and patients.

\section{MATERIALS AND METHODS}

\section{Phantoms}

A phantom study was performed using the ACR phantom and Quantitative Imaging Biomarker Alliance (QIBA) DW-MRI icewater phantom to assess GD and ADC consistencies, respectively. The ACR MRI phantom diameter is $190 \mathrm{~mm}$ and the length is $148 \mathrm{~mm}$, and it is filled with a solution of $10 \mathrm{mM} \mathrm{NiCl} \mathrm{N}_{2}$ and $75 \mathrm{mM} \mathrm{NaCl}$. The phantom was kept in the scanner room at least 24 hours to achieve thermal equilibrium prior to scan; the standard scanning protocol was conducted as specified by ACR (https://accreditationsupport.acr.org/support/solutions/articles/ 11000061035-large-phantom-testing-mri-revised-12-12-19-). Slice \#5 of the image set acquired using the standard protocol specified by ACR is used as a reference image and is compared with the matched slice data obtained by $\mathrm{T}_{2}$-weighted $\left(\mathrm{T}_{2} \mathrm{w}\right) \mathrm{MRI}$. Geometric dimensions and landmark coordinates of the ACR phantom were measured to assess distortion.

The quantitative diffusion phantom (High Precision Devices, Inc, Boulder, CO) developed by the National Institute of Standards and Technology/Radiological Society of North America-QIBA consists of \# 1-13 vials filled with varying concentrations of polyvinylpyrrolidone (PVP) in an aqueous solution (46). The phantom was specifically designed for quantitatively mapping isotropic Gaussian diffusion of water molecules and generating physiologically relevant $\mathrm{ADC}$ values. The distribution of PVP concentrations in the phantom is as follows: $0 \%$ (vials 1, 2, and 8), 10\% (vials 3 and 9), 20\% (vials 4 and 10), 30\% (vials 5 and 11), 40\% (vials 6 and 12), and 50\% (vials 7 and 13). In this study, seven vials included the vial at the isocenter, and six inner vials were selected for ADC measurement on the central slice; consistency was compared between methods. The space between the vials within the phantom was filled with an ice-water bath 24 hours before scanning to eliminate thermal variability at the scanner location and time points in $\mathrm{ADC}$ measurements by maintaining the inner phantom temperature at $0^{\circ} \mathrm{C}$. To study the distortion caused by the air-filled region while using the EPI-based DW-MRI method, a small portion of ice water was removed just before data acquisition on the MRI scanner to create an air-filled space on the anterior portion around vials \#5 and 6 to mimic the conditions typically found in biological tissues in the $\mathrm{HN}$ region $(28,33)$.

\section{Patient Cohort}

The institutional review board approved this Health Insurance Portability and Accountability Act-compliant prospective study for patients $(n=11)$ with cancer in the $\mathrm{HN}$ region including the parotid gland $(n=6)$, skull base $(n=3)$, thyroid gland $(n=1)$, and nasal cavity $(n=1)$. Written informed consent was obtained from all eligible patients with a median age of 60 years (range, 36-84 years; men, 6 and women, 5) who enrolled between October 2018 and October 2019. All patients underwent conventional MRI with additional DW-MRI sequences irrespective of the treatment time point (pretreatment $[n=1]$, intra-treatment $[n=1]$, post-treatment $[n=9])$.

\section{DW-MRI Data Acquisition}

This study was performed on a GE Signa 3T, a 70-cm-bore scanner (Discovery MR750w, GE Healthcare, Waukesha). For ACR and QIBA ice-water phantoms, an 8-channel HD brain array coil was used based on ACR and QIBA/RSNA recommendations (46). HN phase array coil (GE Healthcare, Waukesha) was used for 
patient MRI data acquisition. The same acquisition parameters were used for both phantom and patient studies.

1. $T_{2} w$ fast spin echo anatomical imaging: FOV: $18-22 \times 18-$ $22 \mathrm{~cm}$; matrix, $320 \times 224$; repetition time (TR)/TE, 25004861/102 milliseconds; band width (BW), $83 \mathrm{kHz}$; slice thickness, $3 \mathrm{~mm}$; number of slices, 37-50; number of excitation (NEX), 2; echo train length, 19-21; and total scan time: 1:14-3:10 (minute:second).

2. SS-DWEPI: FOV, $26 \times 26 \mathrm{~cm}$; matrix, $128 \times 128$; TR/TE, 6000/73 milliseconds; single spin echo; number of shot, 1 ; array coil spatial sensitivity encoding (ASSET) acceleration, 2; BW, $250 \mathrm{kHz}$; slice thickness, $5 \mathrm{~mm}$; number of slices, 14-24; b-value, $0 \mathrm{~s} / \mathrm{mm}^{2}$ (2 NEX) and $1000 \mathrm{~s} / \mathrm{mm}^{2}(12$ $\mathrm{NEX);} \mathrm{diffusion} \mathrm{encoding,} \mathrm{all} 3$ directions; and scan time, 1:42 (minute:second).

3. MUSE-DWEPI: FOV, $26 \times 26 \mathrm{~cm}$; matrix, $128 \times 130$; TR/ TE, 6000/74 milliseconds; single spin echo; number of shot, 2; ASSET acceleration, 1.5; BW, $250 \mathrm{kHz}$; slice thickness, $5 \mathrm{~mm}$; number of slices, 14-26; b-value, $0 \mathrm{~s} / \mathrm{mm}^{2}$ (2 NEX) and $1000 \mathrm{~s} / \mathrm{mm}^{2}$ (12 NEX); diffusion encoding, all 3 directions; and scan time, 3:24 (minute:second). The RPG option on the control variable panel was turned on such that images with and without RPG were generated.

\section{DW-MRI Data Analysis}

Statistical Analysis for Phantom Data. The GD calculation was performed on the ACR phantom $b=0 \mathrm{~s} / \mathrm{mm}^{2}$ and $b=1000$ $\mathrm{s} / \mathrm{mm}^{2}$ images obtained from SS-DWEPI, MUSE-DWEPI, and MUSE-DWEPI with RPG by comparing these with the reference $\mathrm{T}_{2} \mathrm{~W}$ image separately. On the GD section of the ACR phantom, four differently spaced $\left(0^{\circ}, 60^{\circ}, 90^{\circ}\right.$, and $120^{\circ}$ represented by red, yellow, green, and blue, respectively) radial lines were used to measure the length between two points selected on the grid as shown in Figure 1. The length of each line was measured, and the percentage of GD (\% GD) was calculated using equation (2).

$$
\% G D=\frac{D_{R}-D_{A}}{D_{R}} \times 100
$$

where $D_{R}$ is the actual dimension on the reference image $\left(\mathrm{T}_{2} \mathrm{~W}\right.$ image), and $D_{A}$ is the measured dimension on the DW-MRI image.

Similarly, three control points (CP) (red, isocenter section; yellow and green, off-isocenter section on edges of the center horizontal grid line) on the same GD section were used to analyze the vector shift, as shown in Figure 1. The relative displacements were calculated between the DW-MRI sequences and the $\mathrm{T}_{2} \mathrm{~W}$ reference image. The overall distortion levels were quantitatively measured using the mean value with standard deviation (SD), and the root-mean-square errors (RMSEs) of the relative displacements were calculated using equation (3).

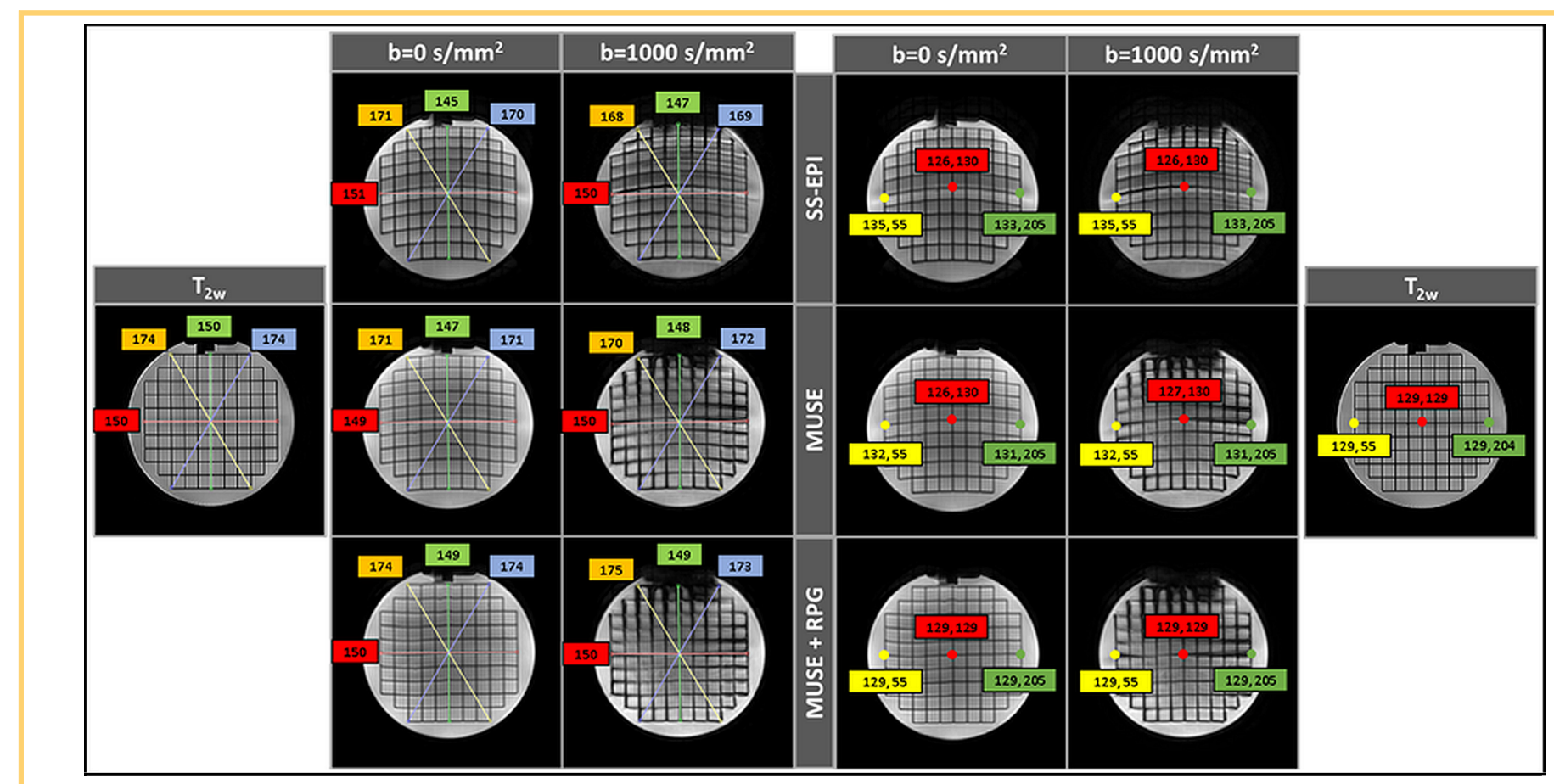

Figure 1. Geometric distortion (GD) measurement on the three diffusion-weighted-magnetic resonance imaging (DWMRI methods, namely, single-shot diffusion-weighted echo planar imaging (SS-DWEPI), multiplexed sensitivity encoding (MUSE)-DWEPI, and MUSE-DWEPI with reverse-polarity gradient (RPG) compared with spin echo (SE)-based $\mathrm{T}_{2}$-weighted $\left(\mathrm{T}_{2} \mathrm{w}\right.$ ) image on a GD section of American College of Radiology (ACR) phantom. Four colored (red, yellow, green, and blue) lines drawn at an angle $\left(0^{\circ}, 60^{\circ}, 90^{\circ}\right.$, and $\left.120^{\circ}\right)$ to measure the distance $(\mathrm{mm})$ between the two points selected on the edge of the grid to check the percentage of GD. Three Control Points (CPs [represented in yellow, red, and green]) are marked on the center horizontal line of the grid to measure the CP displacement. 
Table 1. Summary of Geometric Distortion Analysis Performed on American College of Radiology Phantom

\begin{tabular}{|c|c|c|c|c|c|c|}
\hline \multicolumn{10}{|c|}{ Analysis Performed on American College of Radiology Phantom } \\
\hline \\
\hline
\end{tabular}

$$
R M S E=\sqrt{\frac{\sum_{i=1}^{n} \Delta D_{i}^{2}}{n}}
$$

where $i$ is the index of control points, $n$ is the total number of control points, and $\Delta D_{i}$ is the relative displacement of an $i^{\text {th }}$ control point.

ADC maps for the QIBA ice-water phantom were generated using the vendor-provided software. A circular region of interest
(ROI) was selected manually on the ADC map from these three methods (SS-DWEPI, MUSE-DWEPI, and MUSE-DWEPI with $\mathrm{RPG})$ for each vial from \#1-7. The number of pixels in the ROI was 203.

Qualitative and Quantitative Assessment for Patient Cohort. The GD was evaluated in the HN region for the 11 patient data sets. The $\mathrm{T}_{1} \mathrm{~W}$ postcontrast and $\mathrm{T}_{2} \mathrm{~W}$ images were used as reference images to compare the $\mathrm{b}=0 \mathrm{~s} / \mathrm{mm}^{2}$ and $\mathrm{b}=1000 \mathrm{~s} / \mathrm{mm}^{2}$ images
Figure 2. Apparent diffusion coefficient (ADC) values in the selected seven vials (labeled on $\mathrm{T}_{2} \mathrm{~W}$ image) of the Quantitative Imaging Biomarker Alliance (QIBA) DW-MRI ice-water phantom. GD in both $b=0 \mathrm{~s} / \mathrm{mm}^{2}$ and $b=1000 \mathrm{~s} / \mathrm{mm}^{2}$ images of all the three DW-MRI methods (SS-DWEPI, MUSEDWEPI, and MUSE-DWEPI with RPG) and the effect of same in the ADC maps (A). Standard deviation (SD) of the mean ADC value in the selected seven vials from these three DW-MRI methods (B).

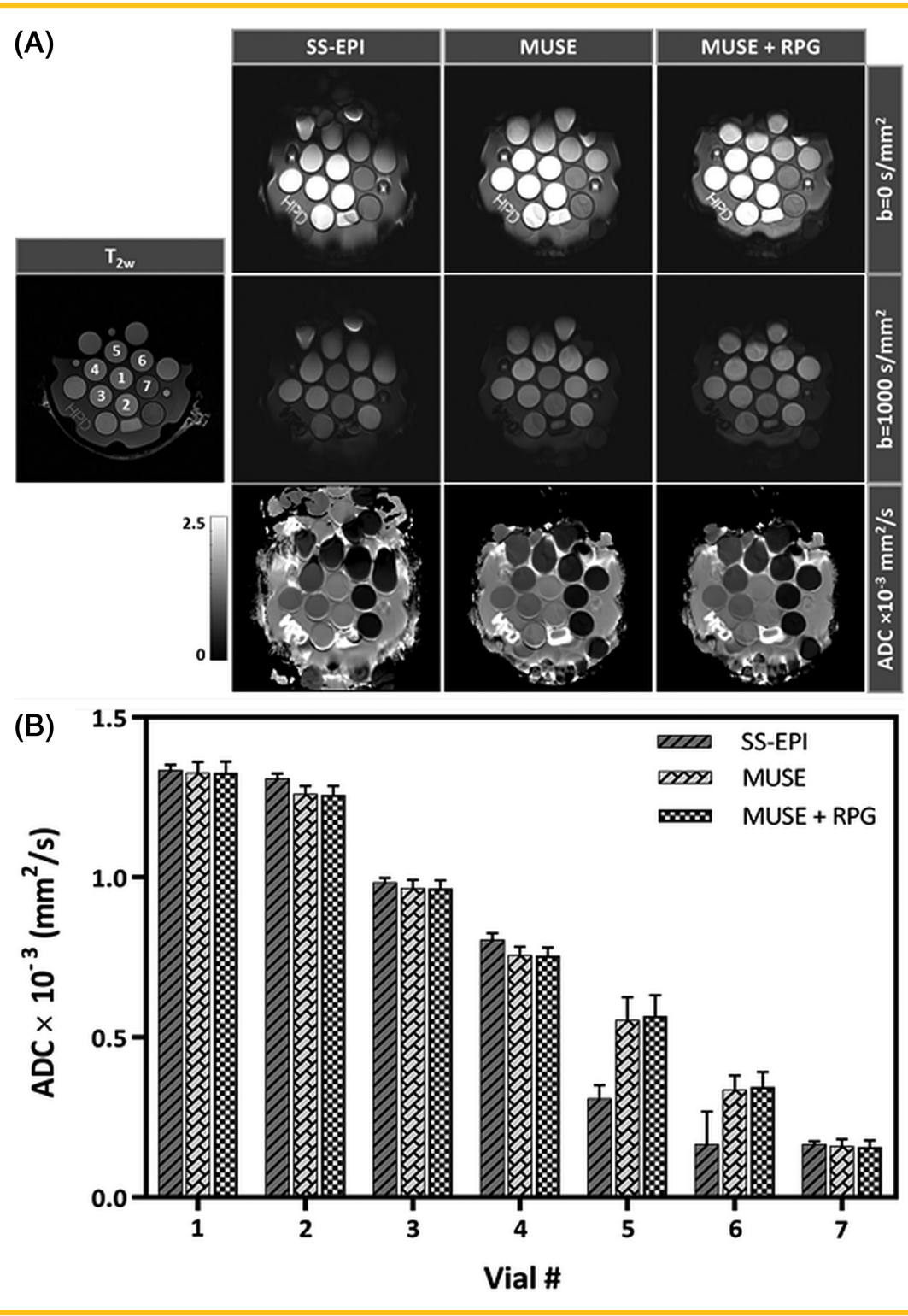




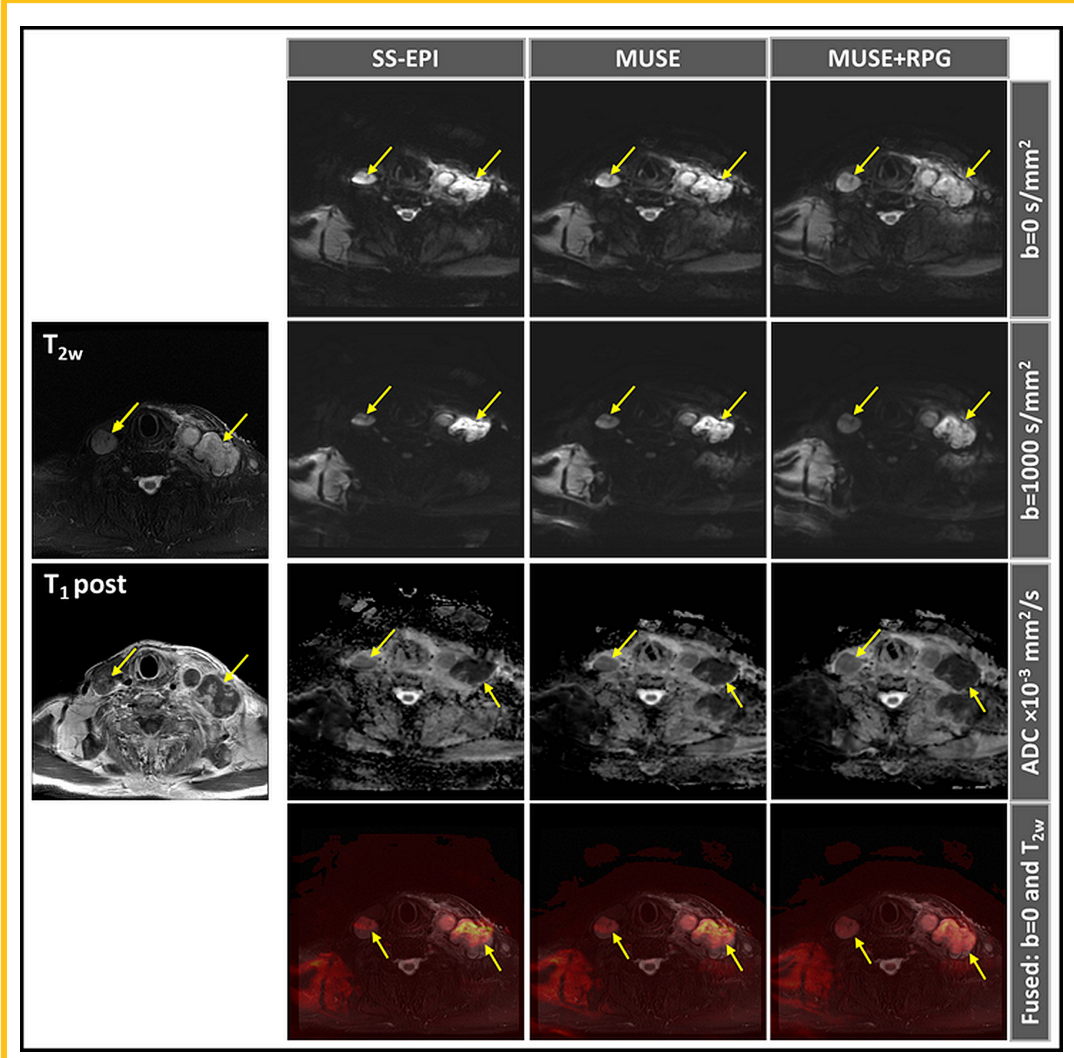

Figure 3. Demonstration of reduction in GD with MUSE-DWEPI and MUSE-DWEPI with RPG in a 76-year-old male patient. $T_{2} \mathrm{w}$ and $\mathrm{T}_{1} \mathrm{w}$ contrastenhanced images are used as reference images for anatomical comparison with the three DW-MRI methods (SS-DWEPI, MUSE-DWEPI, and MUSEDWEPI with RPG). Improved spatial accuracy was observed in the metastatic supraclavicular lymph node (yellow arrow) on either side using MUSEDWEPI with RPG. The fusion between the $\mathrm{T}_{2} \mathrm{w}$ reference image and $b=0 \mathrm{~s} / \mathrm{mm}^{2}$ image of all the three methods are in the $4^{\text {th }}$ row. Marked distortion was observed in SS-DWEPI, and improved geometric accuracy and lesion definition were observed in MUSE-DWEPI and MUSE-DWEPI with RPG.

along with the corresponding ADC maps (generated using the vendor software on the scanner) of these three different DW-MRI methods (SS-DWEPI, MUSE-DWEPI, and MUSE-DWEPI with RPG); the values were measured on the central slices of both the tumor and masseter muscle. An experienced neuroradiologist evaluated all the images and placed ROIs on tumor tissue and healthy tissue (masseter muscle) for reference. ADC values with SDs were measured for the healthy masseter muscle reference region for all patients, except one patient who had no healthy masseter muscle on either the right or left side as per the neuroradiologist's assessment. ADC values for the tumor tissue ROIs were analyzed separately for each patient for these three DWMRI methods. In total, six patients were selected for the tumor analysis because the remaining patients had no measurable posttreatment tumors. The same neuroradiologist rated the image quality based on three criterion; anatomic detail, lack of susceptibility-induced artifacts, and perceived clinical utility. The 5-point scale used in the present study was as follows: 1, nondiagnostic; 2, poor; 3, satisfactory; 4, good; and 5, excellent. All assessments were performed irrespective of the treatment time point and location of the tumor in the $\mathrm{HN}$ region.

\section{Statistical Analysis for the Patient Data}

The correlation coefficients (CCs) between the $\mathrm{T}_{2} \mathrm{~W}$ and DW-MRI image $\left(b=1000 \mathrm{~s} / \mathrm{mm}^{2}\right)$ signal intensities were calculated to measure geometric accuracy (structural similarity) in each method. A binary mask (with ones and zeros) was manually generated by selecting the entire anatomical region and multiplied with the images to avoid the background information in the
CC calculation. These binary masks had ones in the selected anatomical region and zeros in the background. The CCs were calculated using the standard inbuilt function in MATLAB ${ }^{\circledR}$ (The MathWorks Inc., Boston, MA). The ADCs and CCs calculated for the ROIs were averaged to report the mean and SD. A nonparametric Wilcoxon signed-rank (WSR) test was performed to assess the difference between each pair of the methods (SS-DWEPI, MUSE-DWEPI, and MUSE-DWEPI with RPG). Kruskal-Wallis test was used to compare ADC values and CCs obtained with the three methods. Bonferroni correction was applied to address the multiple comparisons between the radiological evaluation criterion (anatomical details, lack of artifact, and perceived clinical utility) and the three different DW-MRI methods. This analysis leads to 12 independent tests, and the resultant $P$-values were corrected using the Bonferroni correction. A $P$-value $<.05$ was considered statistically significant, and an adjusted $P$-value $<$ $.0042(0.05 / 12)$ was regarded as statistically significant after Bonferroni correction. All statistical analyses were performed on GraphPad Prism ${ }^{\circledR}$ version 7 (San Diego, CA).

\section{RESULTS}

Figure 1 shows radial lines and control points plotted on top of the DW-MRI images $\left(b=0\right.$ and $\left.b=1000 \mathrm{~s} / \mathrm{mm}^{2}\right)$ and reference $\mathrm{T}_{2} \mathrm{~W}$ image of ACR phantom. A GD appeared reduced in MUSEDWEPI with RPG compared with that in MUSE-DWEPI and SSDWEPI. Table 1 summarizes the percentage of GD measurements for these three DW-MRI methods. A GD of $0.17 \%$ was observed for the $b=0 \mathrm{~s} / \mathrm{mm}^{2}$ image of MUSE-DWEPI with the RPG method along the phase-encoding directions compared with 
Figure 4. Demonstration of reduction in GD near the air-tissue interface in the head and neck (HN) region (post-thyroidectomy) of a 76-year-old male patient by use of MUSE-DWEPI with RPG. $T_{2} \mathrm{w}$ and $\mathrm{T}_{1} \mathrm{w}$ contrast-enhanced images were used as reference images for anatomical comparison with the three DW-MRI methods (SS-DWEPI, MUSE-DWEPI, and MUSE-DWEPI with RPG). Improved spatial accuracy was observed in the metastatic lymph node in the left thyroid bed (yellow arrow) using MUSEDWEPI with RPG. The fusion between the $\mathrm{T}_{2} \mathrm{~W}$ reference image and $b=0 \mathrm{~s} / \mathrm{mm}^{2}$ image of all the three methods is in the $4^{\text {th }}$ row. Marked geometric accuracy and lesion definition were observed in MUSEDWEPI with RPG

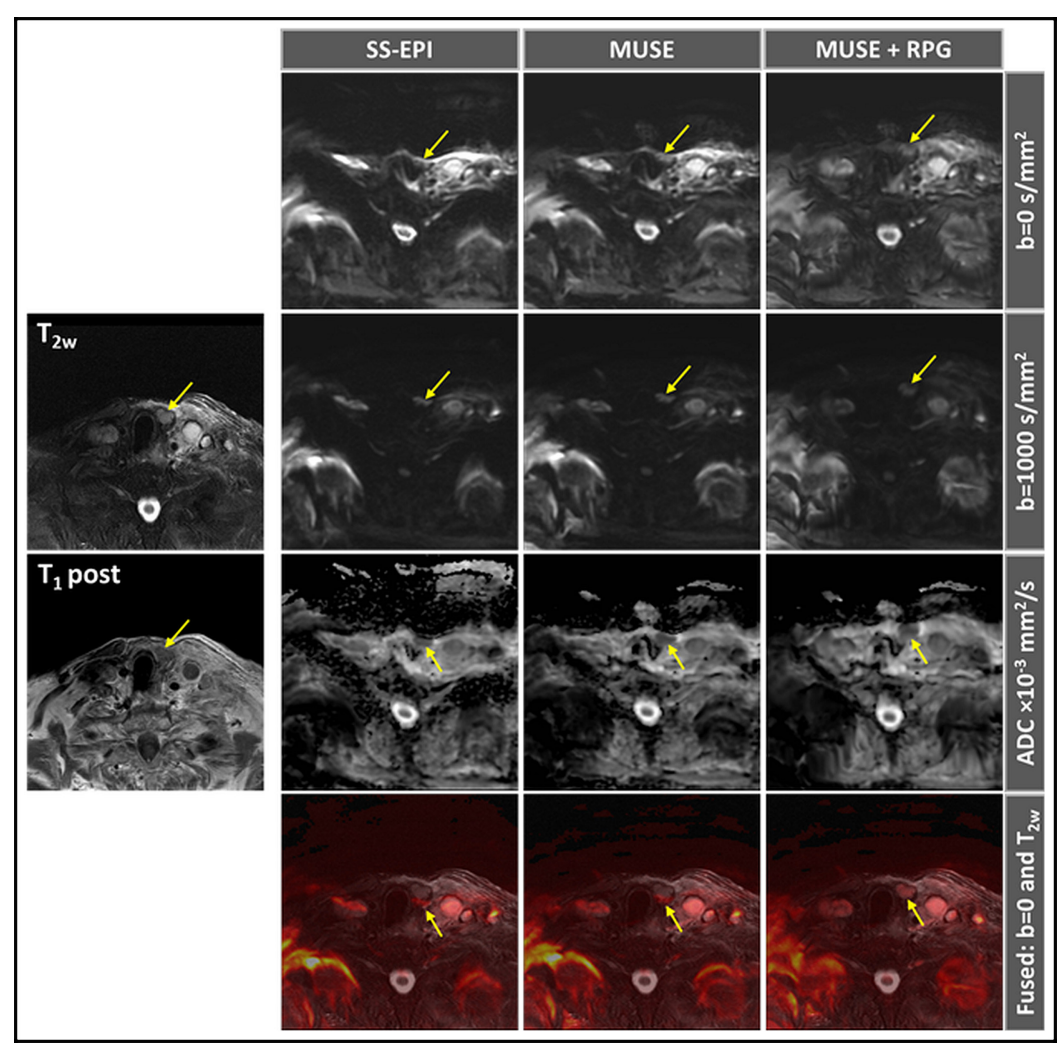

the left thyroid bed (post-thyroidectomy) using these three DW-MRI methods and MUSE-DWEPI with RPG. Figure 5 shows the mean $\mathrm{ADC}$ values measured for healthy masseter muscle with no significant difference between the three DW-MRI methods, that is, ADC $\times$ $\left(10^{-3} \mathrm{~mm}^{2} / \mathrm{s}\right)=1.620( \pm 0.229), 1.584( \pm 0.184)$, and $1.604( \pm 0.189)$ SS-DWEPI; $3.37 \mathrm{~mm}$ and $3.33( \pm 0.58) \mathrm{mm}$, respectively, for MUSE-DWEPI; and $0.58 \mathrm{~mm}$ and $0.33( \pm 0.58) \mathrm{mm}$, respectively, for MUSE-DWEPI with RPG. Figure 2 depicts the distortion in the DW images and the ADC map. Distortion is related to the amount of air surrounding the vials, and it is visible in the uppermost portion of the DW images of these three methods. GD appeared reduced in MUSE-DWEPI with RPG compared with MUSEDWEPI and SS-DWEPI. In vials \#5 and \#6 of the QIBA ice-water phantom, the $\mathrm{ADC} \times\left(10^{-3} \mathrm{~mm}^{2} / \mathrm{s}\right)$ values were calculated using SS-DWEPI (0.311 [ \pm 0.040$]$ and 0.166 [ \pm 0.102$]$, respectively), MUSE-DWEPI (0.554 [ \pm 0.071$]$ and 0.337 [ \pm 0.043$]$, respectively), and MUSE-DWEPI with RPG (0.567 [ \pm 0.065$]$ and 0.346 [ \pm 0.046$]$, respectively). The $\mathrm{ADC}$ values measured using SSDWEPI showed substantial variation compared with MUSEDWEPI or MUSE-DWEPI with RPG. The percentage of the difference calculated for the ADC values measured for vials \#5 and \#6 were 56\% and 68\%, respectively, between SS-DWEPI and MUSE-DWEPI, 58\% and 70\%, respectively, between SS-DWEPI and MUSE-DWEPI with RPG compared with 23\% and 26\%, respectively, between MUSE-DWEPI and MUSE-DWEPI with RPG.

Figure 3 shows the subsequent improvement in the spatial accuracy of MUSE-DWEPI with RPG in the metastatic bilateral neck lymph nodes. Figure 4 depicts the metastatic lymph node in

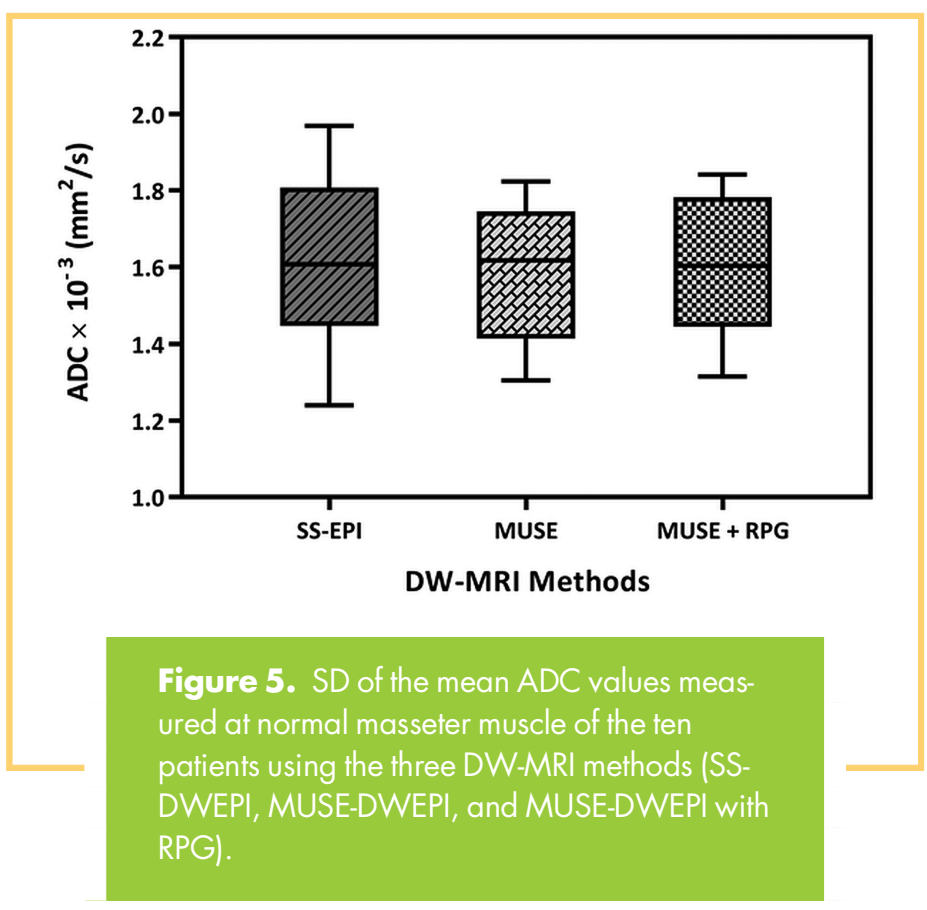


Table 2. Summary of Statistical Analysis Performed on Apparent Diffusion Coefficient Values Obtained Using SS DWEPI, MUSE-DWEPI, and MUSE-DWEPI with RPG

\begin{tabular}{|l|c|c|c|c|}
\hline \multicolumn{1}{|c|}{ ROI } & $\begin{array}{c}\text { Wilcoxon Signed-Rank Test } \\
\text { SS-DWEPI and } \\
\text { MUSE-DWEPI }\end{array}$ & $\begin{array}{c}\text { SS-DWEPI and MUSE- } \\
\text { DWEPI with RPG }\end{array}$ & $\begin{array}{c}\text { MUSE-DWEPI and MUSE- } \\
\text { DWEPI with RPG }\end{array}$ & $\begin{array}{c}\text { Kruskal-Wallis } \\
\text { Test }\end{array}$ \\
\hline Masseter muscle & .193 & .432 & .074 & .815 \\
\hline Tumor & .063 & .999 & .438 & .966 \\
\hline
\end{tabular}

All are $P$-values.

for the SS-DWEPI, MUSE-DWEPI, and MUSE-DWEPI with RPG, respectively. A higher \pm SD was observed for the SS-DWEPI method. Results of the WSR and KW tests performed on the ADC values measured at the masseter muscle and tumor region are summarized in Table 2. Figure 6 shows the SD of the mean ADC values measured for the tumors located in the HN region. There was measurable disease in 6 patients (pretreatment $[n=1]$, intratreatment $[n=1]$, and posttreatment $[n=4])$, while there was no residual disease in 5 patients post treatment. As expected, the range of tumor $\mathrm{ADC}$ values (ADC $\times\left[10^{-3} \mathrm{~mm}^{2} / \mathrm{s}\right]$ from 0.625 to 2.357 ) varied for the 6 patients with disease because of the different treatment time points and locations of the tumor in the HN. However, no significant difference in ADC values for tumors between the DW-MRI methods $(P$-value $>.05)$ was noted, and the results are summarized in Table 2. Figure 7 shows that MUSE-DWEPI with RPG has the highest correlation $\left(\mathrm{R}^{2}=0.791\right)$ compared with the SS-DWEPI $\left(\mathrm{R}^{2}=0.707\right)$ and MUSE-DWEPI $\left(\mathrm{R}^{2}=0.745\right)$. The WSR and KW tests performed on CC values showed a statistically significant difference $(P$-value $=.001$ for all the three combinations of the WSR test and $P$-value $=.022$ for the KW test). Table 3 shows a higher rating for MUSE-DWEPI with RPG in radiological evaluation

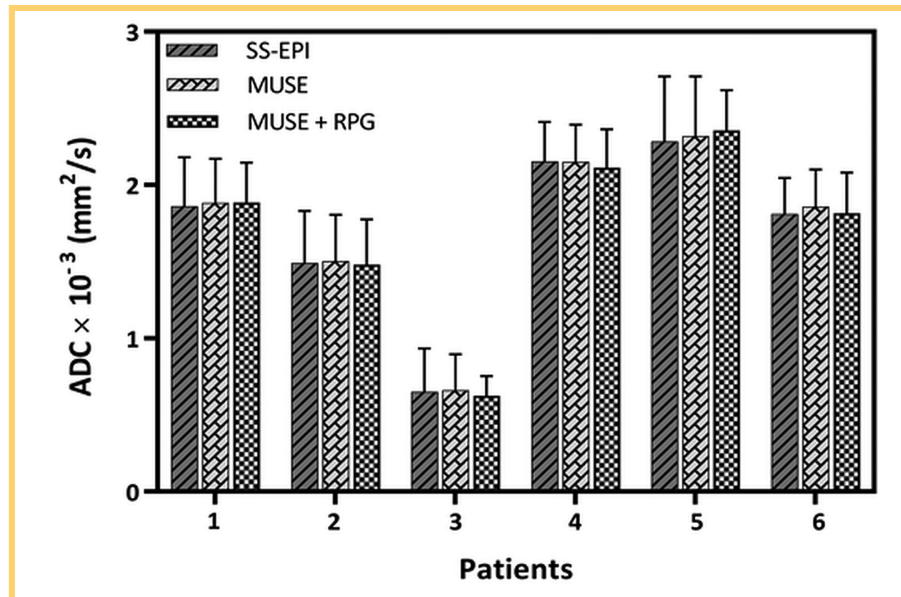

Figure 6. $S D$ of the mean $A D C$ values measured at tumor location for six patients separately using the three DW-MRI methods (SS-DWEPI, MUSE-DWEPI, and MUSE-DWEPI with RPG). criteria (anatomical details, lack of artifacts, and perceived clinical utility) as compared with MUSE-DWEPI and SS-DWEPI. Results of the WSR and KW tests performed on the reader study (based on radiological evaluation criterion) are summarized in Table 4. For the original $P$-values $(<.05)$, the WSR test between pair of the methods (SS-DWEPI and MUSE-DWEPI with RPG) was statistically significant for all the 3 criteria used in the reader study. However, after Bonferroni correction $(P$-value $<$.0042), the anatomical details and clinical utility showed borderline significance. For the original $P$-values, all three KW tests showed statistically significant differences between the three methods. For the corrected $P$-values, the anatomical details and clinical utility showed statistical significance between the three methods.

\section{DISCUSSION}

This is the first study, to the best of our knowledge, to test and evaluate the three DW-MRI methods (SS-DWEPI, MUSE-DWEPI,

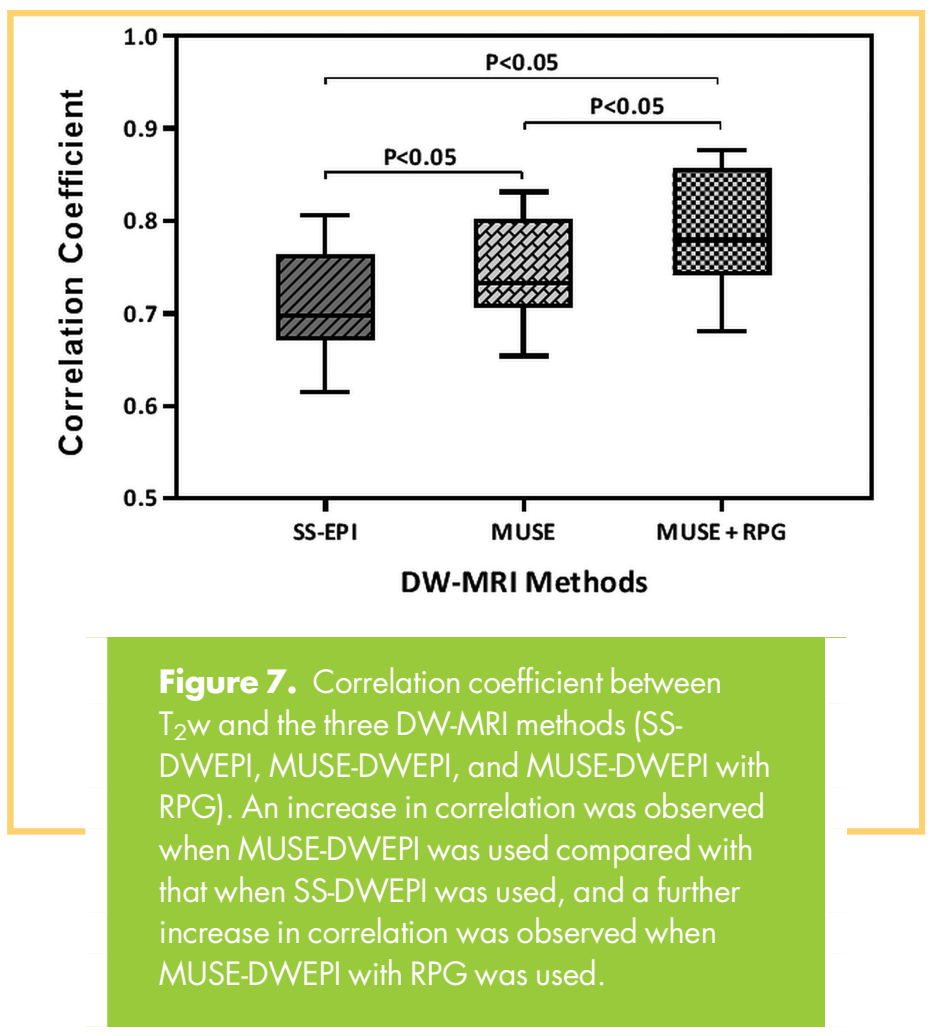


Table 3. Qualitative Rating by a Neuroradiologist Using SS-DWEPI, MUSE-DWEPI, and MUSE-DWEPI with RPG Images

\begin{tabular}{|c|c|c|c|}
\hline Radiological Evaluation Criterion & SS-DWEPI & MUSE-DWEPI & MUSE-DWEPI With RPG \\
\hline Anatomic details & $3.00(0.45)$ & $3.55(0.52)$ & $3.82(0.40)$ \\
\hline Lack of artifact & $3.09(0.30)$ & $3.45(0.52)$ & $3.73(0.47)$ \\
\hline Perceived clinical utility & $3.00(0.45)$ & $3.55(0.52)$ & $3.82(0.45)$ \\
\hline
\end{tabular}

Mean ( \pm SD).

and MUSE-DWEPI with RPG) in both phantoms (ACR and QIBA ice-water) and in the HN region for muscles and tumors. An improved spatial resolution with reduced distortion for MUSE-DWEPI with RPG was observed compared with the other two DW-MRI methods in both phantoms and in the HN region. ADC values obtained for the tumor and the masseter muscle using these three DW-MRI methods showed no significant difference, irrespective of the treatment time point and the location of the tumor in the HN region.

A previous study has reported that MUSE-DWEPI has less distortion than SS-DWEPI in an ACR phantom (43). A maximum value of GD was observed in the SS-DWEPI when compared with MUSE-DWEPI along the phase-encoding direction. ACR phantom results in the present study were consistent with those of this previous study using the SSDWEPI and MUSE-DWEPI (43). The combination of MUSEDWEPI with the RPG method showed a further reduction in distortion in both phantoms and in the HN region.

Recently, MUSE-DWEPI was applied for brain, showing the utility of reduced GD (43). In this study by Chen et al., the most prominent image distortion was along the phase-encoding direction and a maximum GD value of 7\% for SS-DWEPI and 2\% for MUSE-DWEPI. Brain region and target analyses were also involved in this study. They reported higher dice similarity coefficient values and lower Hausdorff distance using MUSE-DWEPI than when using SS-DWEPI ( $P$-value $<$ .01) (43). Similarly, our findings indicate that MUSE-DWEPI is better than SS-DWEPI for distortion reduction in both the ACR phantom and the HN region. HN is a challenging region in the human body to evaluate using DW-MRI owing to airtissue interfaces, such as structures near the paranasal sinuses, nasal cavity, ear canals, and trachea. A major concern in clinically used SS-DWEPI is the magnetic field inhomogeneity, and the extent of distortion is higher in the regions close to air cavities. HN region is a more complex structure compared with the phantoms, and data were separately analyzed, and the results were not compared with each other.

In the present study, ADC values measured for the normal masseter muscles and HN tumors showed no significant difference between these three DW-MRI methods. Similar results were reported for MUSE-DWEPI and readout segmented DWEPI for brain and bladder cancer applications $(43,47)$. Even though there was no significant difference in the ADC values measured between the DW-MRI methods, the qualitative image rating performed by the neuroradiologist showed that MUSE with RPG is superior compared with the other two DW-MRI methods.

A limitation in the preliminary DW-MRI technique assessment study is the small patient cohort. Herein, the three DW-MRI methods were used to study distortion in the HN region in patients irrespective of the treatment time point and tumor location. A larger cohort of patients with cancer in the HN region is needed to validate these findings. Another limitation is that MUSE-DWEPI has a 2-fold longer scan time than SS-DWEPI ( 3:24 [minute:second] for MUSE; 1:42 [minute:second] for SS-DWEPI). However, MUSE offers another opportunity to fully adopt RPG to reduce both $\mathrm{B}_{0}$ - and eddy current-induced distortions with no increase in acquisition time.

In conclusion, our feasibility study shows that MUSEDWEPI with RPG is the superior option for DW-MRI in the HN region because of its ability to reduce distortion compared with conventional SS-DWEPI technique used commonly in clinical studies. There is no significant difference in the ADC values measured using the three different DW-MRI methods. Reduced distortion in MUSE-DWEPI with RPG provides better delineation

\section{Table 4. Summary of Statistical Analysis Performed on Qualitative Rating}

\begin{tabular}{l}
$\begin{array}{l}\text { Radiological } \\
\text { Evaluation Criterion }\end{array}$ \\
\hline Anatomic Details \\
\hline Lack of Artifact \\
\hline Perceived Clinical Utility \\
\hline
\end{tabular}

All are $P$-values.
Wilcoxon Signed-Rank Test

\begin{tabular}{c|c|}
$\begin{array}{c}\text { SS-DWEPI and MUSE- } \\
\text { DWEPI with RPG }\end{array}$ & $\begin{array}{c}\text { MUSE-DWEPI and MUSE- } \\
\text { DWEPI with RPG }\end{array}$ \\
\hline .008 & .250 \\
.016 & .250 \\
.008 & .250 \\
\hline
\end{tabular}

.016
.250
SS-DWEPI and MUSE-DWEPI

.031

.125

.031
Kruskal-

Wallis test .002

.012

.002

-All are P-values. 
of tumors, particularly around the air-tissue interface region, which is typically common in the HN region. This technique has promise in HN for both clinical diagnostic MRI and for treatment planning in radiation oncology.

\section{ACKNOWLEDGMENT}

Equal Contribution: A.K. and M.F. contributed equally to the study.

Co-Senior Authors: V.H. and A.S.D. are co-senior authors.

We would like to thank the MRI technologists for their great efforts in helping to perform the MRI examinations. The U01 CA21 1205 grant supported this work.

\section{REFERENCES}

1. Bray F, Ferlay J, Soerjomataram I, Siegel RL, Torre LA, Jemal A. Global cancer statistics 2018: GLOBOCAN estimates of incidence and mortality worldwide for 36 cancers in 185 countries. CA Cancer J Clin. 2018;68:394-424.

2. King AD, Vlantis AC, Tsang RK, Gary TM, Au AK, Chan CY, Kok SY, Kwok WT, Lui HK, Ahuja AT. Magnetic resonance imaging for the detection of nasopharyngeal carcinoma. AJNR Am J Neuroradiol. 2006;27:1288-1291.

3. Klussmann JP. Head and neck cancer - new insights into a heterogeneous disease. Oncol Res Treat. 2017:40:318-319.

4. King AD, Mo FK, Yu KH, Yeung DK, Zhou H, Bhatia KS, Tse GM, Vlantis AC, Wong JK, Ahuja AT. Squamous cell carcinoma of the head and neck: diffusion-weighted MR imaging for prediction and monitoring of treatment response. Eur Radiol. 2010;20:2213-2220

5. Lenz M, Greess H, Baum U, Dobritz M, Kersting-Sommerhoff B. Oropharynx, oral cavity, floor of the mouth: CT and MRI. Eur J Radiol. 2000;33:203-215.

6. Noda Y, Kanematsu M, Goshima S, Kondo H, Watanabe H, Kawada H, Bae KT. MRI of the thyroid for differential diagnosis of benign thyroid nodules and papillary carcinomas. AJR Am J Roentgenol. 2015;204:W332-W335.

7. Zima AJ, Wesolowski JR, Ibrahim M, Lassig AA, Lassig J, Mukherji SK. Magnetic resonance imaging of oropharyngeal cancer. Top Magn Reson Imaging. 2007; 18:237242

8. Basser PJ, Jones DK. Diffusion-tensor MRI: theory, experimental design and data analysis - a technical review. NMR Biomed. 2002;15:456-467.

9. Sener RN. Diffusion MRI: apparent diffusion coefficient (ADC) values in the norma brain and a classification of brain disorders based on ADC values. Comput Med Imaging Graph. 2001;25:299-326.

10. Razek AA, Elkhamary S, Mousa A. Differentiation between benign and malignant orbital tumors at 3-T diffusion MR-imaging. Neuroradiology. 201 1;53:517-522.

11. Abdel Razek AA, Samir S, Ashmalla GA. Characterization of parotid tumors with dynamic susceptibility contrast perfusion-weighted magnetic resonance imaging and diffusion-weighted MR imaging. J Comput Assist Tomogr. 2017;41:131-136.

12. Abdel Razek AA, Soliman NY, Elkhamary S, Alsharaway MK, Tawfik A. Role of diffusion-weighted MR imaging in cervical lymphadenopathy. Eur Radiol. 2006; 16 : 1468-1477.

13. Srinivasan A, Dvorak R, Perni K, Rohrer S, Mukherii S. Differentiation of benign and malignant pathology in the head and neck using $3 \mathrm{~T}$ apparent diffusion coefficient valves: early experience. AJNR Am J Neuroradiol. 2008;29:40-44.

14. Wang J, Takashima S, Takayama F, Kawakami S, Saito A, Matsushita T, Momose M, Ishiyama T. Head and neck lesions: characterization with diffusion-weighted echo-planar MR imaging. Radiology. 2001;220:621-630.

15. Guo W, Luo D, Lin M, Wu B, Li L, Zhao Y, Yang L, Zhou C. Pretreatment intra-voxel incoherent motion diffusion-weighted imaging (IVIM-DWI) in predicting induction chemotherapy response in locally advanced hypopharyngeal carcinoma. Medicine (Baltimore). 2016;95:e3039.

16. Hatakenaka M, Nakamura K, Yabuuchi H, Shioyama Y, Matsuo Y, Kamitani T, Yonezawa M, Yoshiura T, Nakashima T, Mori M, Honda H. Apparent diffusion coefficient is a prognostic factor of head and neck squamous cell carcinoma treated with radiotherapy. Jpn J Radiol. 2014;32:80-89.

17. Hauser T, Essig M, Jensen A, Laun FB, Munter M, Maier-Hein KH, Stieltjes B. Prediction of treatment response in head and neck carcinomas using IVIM-DWI: evaluation of lymph node metastasis. Eur J Radiol. 2014;83:783-787.

18. Huang WY, Wen JB, Wu G, Yin B, Li JJ, Geng DY. Diffusion-weighted imaging for predicting and monitoring primary central nervous system lymphoma treatment response. AJNR Am J Neuroradiol. 2016;37:2010-2018.

19. Kim S, Loevner L, Quon H, Sherman E, Weinstein G, Kilger A, Poptani H. Diffusionweighted magnetic resonance imaging for predicting and detecting early response to chemoradiation therapy of squamous cell carcinomas of the head and neck. Clin Cancer Res. 2009; 15:986-994.

20. Lu Y, Moreira AL, Hatzoglou V, Stambuk HE, Gonen M, Mazaheri Y, Deasy JO, Shaha AR, Tuttle RM, Shukla-Dave A. Using diffusion-weighted MRI to predict aggressive histological features in papillary thyroid carcinoma: a novel tool for pre-operative risk stratification in thyroid cancer. Thyroid. 2015;25:672-680.
Conflict of Interest: None reported.

Disclosures: No disclosures to report.

21. Ng SH, Lin CY, Chan SC, Lin YC, Yen TC, Liao CT, Chang JT, Ko SF, Wang HM, Chang $\mathrm{CJ}$, Wang JJ. Clinical utility of multimodality imaging with dynamic contrastenhanced MRI, diffusion-weighted MRI, and 18F-FDG PET/CT for the prediction of neck control in oropharyngeal or hypopharyngeal squamous cell carcinoma treated with chemoradiation. PLoS One. 2014;9:e1 15933.

22. Paudyal R, Oh JH, Riaz N, Venigalla P, Li J, Hatzoglou V, Leeman J, Nunez DA, Lu Y, Deasy JO, Lee N, Shukla-Dave A. Intravoxel incoherent motion diffusion-weighted MRI during chemoradiation therapy to characterize and monitor treatment response in human papillomavirus head and neck squamous cell carcinoma. J Magn Reson Imaging. 2017;45:1013-1023.

23. Srinivasan A, Chenevert TL, Dwamena BA, Eisbruch A, Watcharotone K, Myles JD, Mukherii SK. Utility of pretreatment mean apparent diffusion coefficient and apparent diffusion coefficient histograms in prediction of outcome to chemoradiation in head and neck squamous cell carcinoma. J Comput Assist Tomogr. 2012;36:131-137.

24. Desouky S, AboSeif S, Shama S, Gaafar A, Gamaleldin O. Role of dynamic contrast enhanced and diffusion weighted MRI in the differentiation between post treatment changes and recurrent laryngeal cancers. The Egyptian Journal of Radiology and Nuclear Medicine. 2015;46:379-389.

25. Vaid S, Chandorkar A, Atre A, Shah D, Vaid N. Differentiating recurrent tumours from post-treatment changes in head and neck cancers: does diffusion-weighted MRI solve the eternal dilemma? Clin Radiol. 2017;72:74-83.

26. Vandecaveye V, De Keyzer F, Nuyts S, Deraedt K, Dirix P, Hamaekers P, Vander Poorten V, Delaere P, Hermans R. Detection of head and neck squamous cell carcinoma with diffusion weighted MRI after (chemo)radiotherapy: correlation between radiologic and histopathologic findings. Int J Radiat Oncol Biol Phys. 2007;67:960971 .

27. Vandecaveye V, Dirix P, De Keyzer F, de Beeck KO, Vander Poorten V, Roebben I, Nuyts S, Hermans R. Predictive value of diffusion-weighted magnetic resonance imaging during chemoradiotherapy for head and neck squamous cell carcinoma. Eur Radiol. 2010;20:1703-1714.

28. Connolly M, Srinivasan A. Diffusion-weighted imaging in head and neck cancer: technique, limitations, and applications. Magn Reson Imaging Clin N Am. 2018;26: 121-133.

29. Stehling MK, Turner R, Mansfield P. Echo-planar imaging: magnetic resonance imaging in a fraction of a second. Science. $1991 ; 254: 43-50$.

30. Turner R, Le Bihan D, Maier J, Vavrek R, Hedges LK, Pekar J. Echo-planar imaging of intravoxel incoherent motion. Radiology. 1990;177:407-414.

31. Anderson AW, Gore JC. Analysis and correction of motion artifacts in diffusion weighted imaging. Magn Reson Med. 1994;32:379-387.

32. Jezzard $P$, Balaban RS. Correction for geometric distortion in echo planar images from BO field variations. Magn Reson Med. 1995;34:65-73.

33. Gatidis S, Graf H, WeißJ, Stemmer A, Kiefer B, Nikolaou K, Notohamiprodjo M, Martirosian P. Diffusion-weighted echo planar MR imaging of the neck at 3 T using integrated shimming: comparison of MR sequence techniques for reducing artifacts caused by magnetic-field inhomogeneities. MAGMA. 2017;30:57-63.

34. Holland D, Kuperman JM, Dale AM. Efficient correction of inhomogeneous static mag netic field-induced distortion in Echo Planar Imaging. Neuroimage. 2010;50:175183.

35. Atkinson D, Counsell S, Hajnal JV, Batchelor PG, Hill DL, Larkman DJ. Nonlinear phase correction of navigated multi-coil diffusion images. Magn Reson Med. 2006;56:1135-1139.

36. Atkinson D, Porter DA, Hill DL, Calamante F, Connelly A. Sampling and reconstruction effects due to motion in diffusion-weighted interleaved echo planar imaging. Magn Reson Med. 2000;44:101-109.

37. Bammer R, Stollberger R, Augustin M, Simbrunner J, Offenbacher $H$, Kooijman $H$, Ropele S, Kapeller P, Wach P, Ebner F, Fazekas F. Diffusion-weighted imaging with navigated interleaved echo-planar imaging and a conventional gradient system. Radiology. 1999;211:799-806.

38. Butts K, de Crespigny A, Pauly JM, Moseley M. Diffusion-weighted interleaved echoplanar imaging with a pair of orthogonal navigator echoes. Magn Reson Med. 1996;35:763-770. 
39. Porter DA, Heidemann RM. High resolution diffusion-weighted imaging using readout-segmented echo-planar imaging, parallel imaging and a two-dimensional navigator-based reacquisition. Magn Reson Med. 2009;62:468-475.

40. Robson MD, Anderson AW, Gore JC. Diffusion-weighted multiple shot echo planar imaging of humans without navigation. Magn Reson Med. 1997;38:82-88.

41. Chen NK, Guidon A, Chang HC, Song AW. A robust multi-shot scan strategy for high-resolution diffusion weighted MRI enabled by multiplexed sensitivity-encoding (MUSE). Neuroimage. 2013;72:41-47.

42. Pruessmann KP, Weiger M, Scheidegger MB, Boesiger P. SENSE: sensitivity encoding for fast MRI. Magn Reson Med. 1999;42:952-962.

43. Chen $X$, Zhang $Y$, Cao $Y$, Sun $R$, Huang $P, X u Y$, Wang W, Feng $Q$, Xiao J, Yi J, Li Y, Dai J. A feasible study on using multiplexed sensitivity-encoding to reduce geometric distortion in diffusion-weighted echo planar imaging. Magn Reson Imaging. 2018:54:153-159.
44. Morgan PS, Bowtell RW, Mclntyre DJ, Worthington BS. Correction of spatial distortion in EPI due to inhomogeneous static magnetic fields using the reversed gradient method. J Magn Reson Imaging. 2004; 19:499-507.

45. Rakow-Penner RA, White NS, Margolis DJ, Parsons JK, Schenker-Ahmed N, Kuperman JM, Bartsch H, Choi HW, Bradley WG, Shabaik A, Huang J, Liss MA, Marks L, Kane CJ, Reiter RE, Raman SS, Karow DS, Dale AM. Prostate diffusion imaging with distortion correction. Magn Reson Imaging. 2015;33: $1178-1181$.

46. Boss MA, Chenevert T, Jackson E, Attariwala R, Shukla-Dave A, Amaro E, Shiroishi $M, W u O$, Malyarenko D, Kirsch J. Multicenter study of reproducibility of wide range of $A D C$ at $0^{\circ} \mathrm{C}$. In: RSNA Annual Meeting; 2015; Chicago, IL.

47. Chen $\mathrm{H}, \mathrm{Chen} \mathrm{L}$, Liu F, Lu J, Xu C, Wang L. Diffusion-weighted magnetic resonance imaging in bladder cancer: comparison of readout-segmented and single-shot EPI techniques. Cancer Imaging. 2019;19:59. 